\title{
FUNCTIONS OF CONDITIONALLY NEGATIVE TYPE ON KAZHDAN GROUPS
}

\author{
ANNABEL DEUTSCH AND A. GUYAN ROBERTSON
}

(Communicated by Palle E. T. Jorgensen)

\begin{abstract}
An explicit bound is given for functions of conditionally negative type on Kazhdan groups, in terms of a set of generators and the corresponding Kazhdan constants. This is used to estimate how far a set in an infinite measure space can be translated by the action of a Kazhdan group. Some estimates are given for Kazhdan constants.
\end{abstract}

\section{INTRODUCTION}

The groups $G$ considered in this paper are always assumed to be locally compact with a countable base. If such a group has the discrete topology, it will be denoted by $\Gamma$. Property (T) was introduced by Kazhdan in 1966. His definition says that $G$ has Property (T) if the trivial representation is isolated in the unitary dual of $G$. This concept has been the subject of considerable recent interest, and much information concerning it has been collected in [HaV], to which we refer for further details.

Among several characterizations of Property $(\mathrm{T})$ for a group $G$ we consider the following:

$G$ is compactly generated and for any compact generating set $K$, there exists $\varepsilon>0$, such that if $\pi: G \rightarrow \mathscr{U}(\mathscr{H})$ is a unitary representation of $G$ on a Hilbert space $\mathscr{H}$, and $\zeta \in \mathscr{H}$ with $\|\zeta\|=1$ is such that

$$
\sup _{g \in K}\|\pi(g) \zeta-\zeta\|<\varepsilon,
$$

then $\pi$ has a nonzero invariant vector. The largest value of $\varepsilon$ corresponding to a given $G$ and $K$ is the Kazhdan constant $\kappa(G, K)$ [HRV].

Another property equivalent to Property $(\mathrm{T})$ is the absence of unbounded conditionally negative type functions [ HaV, Chapter 5 , Theorem 20]. This result was proved in [Gui, Del], and, independently, in [AkW].

We shall establish a bound on conditionally negative type functions on a Kazhdan group $G$ in terms of the supremum on a compact generating set $K$ and the Kazhdan constant $\kappa(G, K)$ (Theorem 2 and Corollary 3 below). This says roughly that a conditionally negative type function cannot grow too fast outside $K$.

Received by the editors May 7, 1993.

1991 Mathematics Subject Classification. Primary 22D10. 
Conditionally negative type functions are more usually referred to as continuous negative definite functions in English. We use the European terminology, stressing that a conditionally negative type function is not simply the negative analogue of a positive type (continuous positive definite) function. We refer to [HaV, Chapter 5] for an introduction to these functions.

We show (Corollary 5 ) that for sufficiently large compact generating sets $K$ the Kazhdan constants are greater than $1-\varepsilon$ for any $\varepsilon>0$. This leads to a further refinement of Theorem 2. Then we give an application to Kazhdan groups acting on infinite measure spaces to obtain a bound on how far sets can be translated by the group action (Theorem 7). A related result on translates of functions is given (Proposition 9). Finally we obtain an estimate for Kazhdan constants for paradoxical decompositions.

\section{A BOUND FOR FUNCTIONS OF CONDITIONALLY NEGATIVE TYPE ON KAZHDAN GROUPS}

Consider a real-valued function, $\psi$, of conditionally negative type on a group with Property $(\mathrm{T})$. We know from $[\mathrm{HaV}, 5.20]$ that $\psi$ is bounded. Here we find a bound for $\psi$ as a function of the supremum of $\psi$ on a compact generating set and the Kazhdan constant for that generating set.

First we need some terminology.

Let $\pi$ be a unitary representation of a group $G$. Suppose $K$ is a compact subset of $G$ and $\varepsilon>0$. A unit vector $\zeta \in \mathscr{H}_{\pi}$ is $(\varepsilon, K)$-invariant if

$$
\sup _{g \in K}\|\pi(g) \zeta-\zeta\|<\varepsilon .
$$

Thus $G$ has Property (T) if and only if there is a number $\varepsilon>0$ such that each unitary representation of $G$ which has an $(\varepsilon, K)$-invariant unit vector also has a nonzero invariant vector.

We begin with a lemma, the proof of which is taken from the proof of $[\mathrm{HaV}$, 1.16].

Lemma 1. Let $G$ be a group with Property $(\mathrm{T}), K$ a compact generating set for $G$, and $\delta$ a positive number. Let $\varepsilon=\kappa(G, K)$. If $\pi$ is a representation of $G$ on a Hilbert space $\mathscr{H}$, and $\zeta \in \mathscr{H}$ is an $(\varepsilon \delta, K)$-invariant unit vector for $\pi$, then $\|\pi(g) \zeta-\zeta\|<2 \delta$ for every $g \in G$.

Proof. Let $\pi: G \rightarrow \mathscr{U}(\mathscr{H})$ be a representation with $(\varepsilon \delta, K)$-invariant unit vector $\zeta$. If $\delta>1$, then $\|\pi(g) \zeta-\zeta\| \leq 2\|\zeta\|<2 \delta$, since $\zeta$ is a unit vector. Suppose $\delta \leq 1$. Then, since $\varepsilon$ is the Kazhdan constant for $G$ and $K, \pi(G)$ has some nonzero invariant vector. Let $\mathscr{H}_{0}$ be the subspace of $\mathscr{H}$ consisting of vectors invariant by $\pi(G)$, and let $\mathscr{H}_{1}$ be the orthogonal complement of $\mathscr{H}_{0}$ in $\mathscr{H}$. Let $\zeta=\zeta_{0}+\zeta_{1}$ be the decomposition of $\zeta$ into components in $\mathscr{H}_{0}$ and $\mathscr{K}_{1}$. If $\zeta_{1}=0$, then $\zeta$ is invariant and the lemma is true. Suppose $\zeta_{1} \neq 0$. Then

$$
\sup _{g \in K}\left\|\pi(g) \frac{\zeta_{1}}{\left\|\zeta_{1}\right\|}-\frac{\zeta_{1}}{\left\|\zeta_{1}\right\|}\right\|=\frac{1}{\left\|\zeta_{1}\right\|} \sup _{g \in K}\|\pi(g) \zeta-\zeta\|<\frac{\varepsilon \delta}{\left\|\zeta_{1}\right\|} .
$$

Since $\mathscr{H}_{1}$ does not contain any nonzero invariant vectors, it cannot contain any $(\varepsilon, K)$-invariant unit vectors and so $\varepsilon \delta /\left\|\zeta_{1}\right\|>\varepsilon$. Hence $\left\|\zeta_{1}\right\|<\delta$. Then, for all $g \in G$,

$$
\|\pi(g) \zeta-\zeta\|=\left\|\pi(g) \zeta_{1}-\zeta_{1}\right\| \leq 2\left\|\zeta_{1}\right\|<2 \delta .
$$


Theorem 2. Let $G$ be a group with Property $(\mathrm{T}), K$ a compact generating set for $G$, and $\psi: G \rightarrow \mathbf{R}$ a conditionally negative type function on $G$. Then for every $g \in G$

$$
0 \leq \psi(g) \leq\left(\frac{2}{\kappa(G, K)}\right)^{2} \sup _{h \in K} \psi(h) .
$$

Proof. It is well known that real-valued conditionally negative type functions take only nonnegative values.

Let $N=\sup \{\psi(g): g \in K\}$; this is finite since $\psi$ is continuous and $K$ is compact. Let $\varepsilon=\kappa(G, K)$.

By Schoenberg's Theorem [HaV, 5.16] for any $t>0$ the function $\phi: G \rightarrow \mathbf{R}$ defined by $\phi(g)=e^{-t \psi(g)}$ is of positive type. Let $(\mathscr{H}, \pi, \zeta)$ be the cyclic representation of $G$ induced by $\phi$. So

$$
e^{-t \psi(g)}=\phi(g)=\langle\pi(g) \zeta \mid \zeta\rangle \quad \forall g \in G .
$$

Notice that $\mathscr{\ell}$ is a real Hilbert space since $\phi$ is real valued and that $\|\zeta\|^{2}=$ $\langle\pi(e) \zeta \mid \zeta\rangle=e^{-t \psi(e)}=1$.

Suppose first that $N=0$. Then, for every $g \in K$,

$$
\langle\pi(g) \zeta \mid \zeta\rangle=e^{-t \psi(g)}=1
$$

and so $\zeta$ is invariant under the action of the generating set $K$. Hence, $\zeta$ is invariant under the action of all of $G$, and so for each $g \in G$

$$
e^{-t \psi(g)}=\langle\pi(g) \zeta \mid \zeta\rangle=1
$$

Hence, $\psi(g)=0=\left(\frac{2}{\varepsilon}\right)^{2} N$ for all $g \in G$.

Now suppose $N>0$. Take $\delta$ such that $0<\delta<1 / \sqrt{2}$ and $t$ such that $0<t<-\frac{1}{N} \ln \left(1-(\varepsilon \delta)^{2} / 2\right)$; noting that $(\varepsilon \delta)^{2} / 2<1$ since $\varepsilon \leq 2$. Then

$$
\begin{aligned}
\sup _{g \in K}\|\pi(g) \zeta-\zeta\|^{2} & =\sup _{g \in K} 2(1-\langle\pi(g) \zeta \mid \zeta\rangle)=\sup _{g \in K} 2\left(1-e^{-t \psi(g)}\right) \\
& =2\left(1-e^{-t N}\right)<(\varepsilon \delta)^{2} .
\end{aligned}
$$

It follows by Lemma 1 that

$$
\|\pi(g) \zeta-\zeta\|<2 \delta \quad \forall g \in G .
$$

Hence, for all $g \in G$

$$
\psi(g)=-\frac{1}{t} \ln \left(1-\left(1-e^{-t \psi(g)}\right)\right)=-\frac{1}{t} \ln \left(1-\frac{1}{2}\|\pi(g) \zeta-\zeta\|^{2}\right)<-\frac{1}{t} \ln \left(1-2 \delta^{2}\right) .
$$

Since this holds for every $t \in\left(0,-\frac{1}{N} \ln \left(1-(\varepsilon \delta)^{2} / 2\right)\right)$, it follows that for all $g \in G$

$$
\psi(g) \leq \frac{N \ln \left(1-2 \delta^{2}\right)}{\ln \left(1-(\varepsilon \delta)^{2} / 2\right)} .
$$

This in turn holds for every $\delta \in(0,1 / \sqrt{2})$, so for every $g \in G$

$$
\psi(g) \leq N \lim _{\delta \rightarrow 0} \frac{\ln \left(1-2 \delta^{2}\right)}{\ln \left(1-(\varepsilon \delta)^{2} / 2\right)}=\left(\frac{2}{\varepsilon}\right)^{2} N .
$$

It is not hard to extend this result to complex-valued functions as follows. 
Corollary 3. Let $G$ be a group with Property (T), $K$ a compact generating set for $G$, and $\psi: G \rightarrow \mathbf{C}$ a conditionally negative type function on $G$. Then for every $g \in G$

$$
|\psi(g)-\psi(e)| \leq 2\left(\frac{2}{\kappa(G, K)}\right)^{4} \sup _{h \in K}|\psi(h)-\psi(e)| .
$$

Proof. This follows from Chapter 5, Corollary 1.9 of [HaV], which says that the function $\psi_{0}: G \rightarrow \mathbf{R}$ defined by $\psi_{0}(g)=\mathfrak{R}\left([\psi(g)-\psi(e)]^{1 / 2}\right)$ is of conditionally negative type (in the real-valued sense) and that $\left|[\psi(g)-\psi(e)]^{1 / 2}\right| \leq \sqrt{2} \psi_{0}(g)$ for all $g \in G$.

\section{KAZHDAN CONSTANTS FOR LARGE GENERATING SETS}

Let $\pi$ be a unitary representation of $\Gamma$ on a Hilbert space $\mathscr{H}$. Recall from [HRV, KaT] that

$$
\kappa(\pi, K)=\inf _{\|\xi\|=1} \max _{k \in K}\|\xi-\pi(k) \xi\| .
$$

Note that $\kappa(\pi, K) \leq 2$. Also $\kappa(\pi, K)=0$ if and only if the trivial representation $\chi_{1}$ is weakly contained in $\pi$ [HRV, Lemma 4].

Assume that $K=K^{-1}$. Let $K_{n}$ denote the set of words of length at most $n$ in $K$. Note that as $n \rightarrow \infty, \kappa\left(\pi, K_{n}\right)$ increases, and hence converges to a limit $\kappa(\pi) \leq 2$.

Theorem 4. If $\kappa(\pi, K)>0$, then $\lim _{n \rightarrow \infty} \kappa\left(\pi, K_{n}\right) \geq 1$.

Proof. The value of $\kappa(\pi, K)$ does not change if the identity element $e$ is added to $K$, so we may assume that $e \in K$.

Fix $n$. Let $\varepsilon>0$, and choose $\xi \in H_{\pi},\|\xi\|=1$, such that

$$
\max _{g \in K_{n}}\|\xi-\pi(g) \xi\|<\kappa\left(\pi, K_{n}\right)+\varepsilon .
$$

Let $h=\frac{1}{|K|} \sum_{k \in K} k \in C^{*}(\Gamma)$. Thus $h=h^{*}$ and $1 \in \operatorname{Sp} h$ [HRV].

Also $h^{n}=\frac{1}{|K|^{n}} \sum k_{1} k_{2} \cdots k_{n}$, where the sum is over all $\left(k_{1}, k_{2}, \ldots, k_{n}\right) \in$ $K^{n}=K \times K \times \cdots \times K$. Now

$$
\begin{aligned}
1 & \leq\left|1-\left\langle\pi\left(h^{n}\right) \xi \mid \xi\right\rangle\right|+\left|\left\langle\pi\left(h^{n}\right) \xi \mid \xi\right\rangle\right| \\
& =\left|\frac{1}{|K|^{n}} \sum_{\left(k_{1}, k_{2}, \ldots, k_{n}\right) \in K^{n}}\left(1-\left\langle\pi\left(k_{1} k_{2} \cdots k_{n}\right) \xi \mid \xi\right\rangle\right)\right|+\left|\left\langle\pi\left(h^{n}\right) \xi \mid \xi\right\rangle\right| \\
& \leq \frac{1}{|K|^{n}} \sum\left|1-\left\langle\pi\left(k_{1} k_{2} \cdots k_{n}\right) \xi \mid \xi\right\rangle\right|+\left\|\pi\left(h^{n}\right)\right\| \\
& \leq \frac{1}{|K|^{n}} \sum\left\|\xi-\pi\left(k_{1} k_{2} \cdots k_{n}\right) \xi\right\|+\left\|\pi\left(h^{n}\right)\right\| \\
& <\kappa\left(\pi, K_{n}\right)+\varepsilon+\left\|\pi\left(h^{n}\right)\right\| .
\end{aligned}
$$

Since $\varepsilon>0$ is arbitrary, we have

$$
\|\pi(h)\|^{n} \geq\left\|\pi\left(h^{n}\right)\right\| \geq 1-\kappa\left(\pi, K_{n}\right) .
$$

Now suppose that $\lim _{n \rightarrow \infty} \kappa\left(\pi, K_{n}\right)=\kappa(\pi)<1$. We show that this implies a contradiction. 
For all $n,\|\pi(h)\|^{n} \geq 1-\kappa(\pi)>0$. Hence $\|\pi(h)\| \geq 1$, and so $\|\pi(h)\|=1$. Since $e \in K$, we know that $-1 \notin \operatorname{Sp} \pi(h)$. Therefore, $1 \in \operatorname{Sp} \pi(h)$. We deduce from [HRV, Proposition I] that $\pi$ weakly contains $\chi_{1}$, i.e., $\kappa(\pi, K)=0$, contrary to assumption.

Recall that $\kappa(\Gamma, K)=\inf _{\pi} \kappa(\pi, K)$, where the infimum is over all unitary representations $\pi$ of $\Gamma$ on a separable Hilbert space which have no nonzero fixed vector.

Corollary 5. Suppose that $\Gamma$ has Kazhdan's Property (T). Then

$$
\sup \left\{\kappa\left(\Gamma, K_{n}\right): n=1,2, \ldots\right\} \geq 1 .
$$

Proof. Since $\Gamma$ has Property $(\mathrm{T}), \kappa(\Gamma, K)>0[\mathrm{HaV}]$. Suppose that the supremum in Corollary 5 is strictly less than $1-\varepsilon$, for some $\varepsilon>0$. Then for each $n=1,2, \ldots$, there is a unitary representation $\pi_{n}$ with no nonzero fixed vector such that $\kappa\left(\pi_{n}, K_{n}\right)<1-\varepsilon$. Let $\pi=\bigoplus_{n \geq 1} \pi_{n}$. For each $n$,

$$
\kappa\left(\pi, K_{n}\right) \leq \kappa\left(\pi_{n}, K_{n}\right)<1-\varepsilon .
$$

This contradicts the theorem and so proves the result.

Remark. If $\Gamma$ is any infinite group, then the supremum in Corollary 5 is less than or equal to $\sqrt{2}$. Just consider the left regular representation $\lambda$ of $\Gamma$, and let $\xi \in l^{2}(\Gamma)$ be the Dirac function at 1 . Then $\|\xi-\lambda(g) \xi\|=\sqrt{2}$ for all $g \in \Gamma$.

From Corollary 5 we can obtain further information regarding the estimates in the previous section. For example,

Corollary 6. Let $\Gamma$ be a discrete Kazhdan group, and let $\varepsilon>0$. Then there exists a sufficiently large generating set $K$ for $\Gamma$ such that, for every conditionally negative type function $\psi: \Gamma \rightarrow \mathbf{R}$,

$$
\sup _{g \in \Gamma}|\psi(g)| \leq(4+\varepsilon) \sup _{g \in K}|\psi(g)| .
$$

Proof. This follows from Corollary 5 and Theorem 2.

\section{A dynamical consequence of Property (T)}

Theorem 7. Let $G$ be a locally compact group with compact generating set $K$. Suppose that there is a measure-preserving action of $G$ on an infinite measure space $(X, \mathscr{B}, \mu)$ and there is a set $S \in \mathscr{B}$ such that $\mu(S \Delta g S)<\infty$ for all $g \in K$. Then $\mu(S \Delta g S)<\infty$ for all $g \in G$. Suppose, moreover, that $G$ has Property (T) and that $\mu(S \Delta g S)$ is a continuous function of $g \in G$. Then $\sup _{g \in G} \mu(S \Delta g S)<\infty$. In fact,

$$
\sup _{g \in G} \mu(S \Delta g S) \leq\left(\frac{2}{\kappa(G, K)}\right)^{2} \sup _{g \in K} \mu(S \Delta g S) .
$$

Proof. In order to prove the first assertion, observe that $\mu(S \Delta g S)=\mu\left(S \Delta g^{-1} S\right)$ for all $g \in G$. Now using the fact that for $g_{1}, g_{2} \in S, S \Delta g_{1} g_{2} S \subset\left(S \Delta g_{1} S\right) \cup$ $\left(g_{1} S \Delta g_{1} g_{2} S\right)$ we obtain by induction that $\mu(S \Delta g S)<\infty$ for all $g \in G$. In order to prove the second assertion, define a function $\eta: G \rightarrow L^{2}(X, \mu)$ by

$$
\eta(g)=\chi_{g S}-\chi_{S}=\chi_{g S-S}-\chi_{S-g S}
$$


where $\chi_{A}$ denotes the characteristic function of a set $A \in \mathscr{B}$. The function $\psi$ defined by

$$
\psi(g)=\|\eta(g)\|_{2}^{2}=\mu(S \Delta g S)
$$

satisfies $\psi(e)=0$. To see that $\psi$ is of conditionally negative definite type, note that

$$
\psi\left(g^{-1} h\right)=\left\|\chi_{h s}-\chi_{g s}\right\|_{2}^{2}=\|\eta(h)-\eta(g)\|^{2} .
$$

Now if $g_{1}, g_{2}, \ldots, g_{n} \in G$ and $\alpha_{1}, \alpha_{2}, \ldots, \alpha_{n}$ are real numbers with $\sum \alpha_{i}=$ 0 , then, writing $\xi_{i}=\eta\left(g_{i}\right)$,

$$
\begin{aligned}
\sum \alpha_{i} \alpha_{j} \psi\left(g_{i}^{-1} g_{j}\right) & =\sum \alpha_{i} \alpha_{j}\left\|\xi_{i}-\xi_{j}\right\|^{2} \\
& =-2 \sum \alpha_{i} \alpha_{j}\left\langle\xi_{i}, \xi_{j}\right\rangle=-2\left\|\sum \alpha_{i} \xi_{i}\right\|^{2} \leq 0 .
\end{aligned}
$$

Since $G$ has Property (T), it follows that $\psi$ is bounded. This proves the result, and the stated inequality follows from Theorem 2.

Remarks. 1. The result can fail if $G$ does not have Property (T). For example, let $G$ be a free group. There exists a homomorphism from $G$ onto $\mathbf{Z}$. Take $X=\mathbf{Z}, \mu=$ counting measure, and $S=\{1,2,3, \ldots\}$, and let $G$ act on $X$ by translation. Then $\mu(S \Delta g S)<\infty$ for all $g \in G$ but $\sup _{g \in G} \mu(S \Delta g S)=\infty$.

2. Continuity of $g \mapsto \mu(S \Delta g S): G \rightarrow \mathbf{R} \cup\{\infty\}$ at the identity element $e$ is sufficient to guarantee continuity on all of $G$. To see this, let $\varepsilon>0$ and choose a neighbourhood $U$ of $e$ in $G$ such that $\mu(S \Delta h S)<\varepsilon$ for all $h \in U$. Then for any $g \in G, g U$ is a neighbourhood of $g$ and, for all $h \in U$,

$$
|\mu(S \Delta g h S)-\mu(S \Delta g S)| \leq \mu(g S \Delta g h S)=\mu(S \Delta h S)<\varepsilon .
$$

Hence, $g \mapsto \mu(S \Delta g S)$ is continuous on $G$.

3. An alternative proof of the boundedness part of Theorem 7 can be given using the cohomological definition of Property (T) $[\mathrm{HaV}$, Chapitre 4] and the cocyle $\eta$ defined above. It follows that $\eta$ is necessarily a coboundary and hence bounded. 7.

Our next result is proved by the same argument as in the proof of Theorem

Proposition 8. Let $G$ be a Kazhdan group with compact generating set $K$ and Haar measure $d h$. For any continuous function $f: G \rightarrow \mathbf{C}$ we have

$$
\sup _{g \in G} \int_{G}|f(h g)-f(h)|^{2} d h \leq\left(\frac{2}{\kappa(G, K)}\right)^{2} \sup _{g \in K} \int_{G}|f(h g)-f(h)|^{2} d h .
$$

Proof. We may assume that the right-hand side of the inequality is finite. Define a function $\eta: G \rightarrow L^{2}(G)$ by $\eta(g)(h)=f(h g)-f(h)$. Now apply the argument as before to the conditionally negative definite function $\psi(g)=\|\eta(g)\|_{2}^{2}$.

Note that we have not assumed that $f \in L^{2}(G)$, as was the case for a similar inequality for a free group [Sak, Lemma 4.3.20].

\section{AN ESTIMATE FOR KAZHDAN CONSTANTS FOR PARADOXICAL DECOMPOSITIONS}

Suppose that a group $\Gamma$ acts on a set $X$ with no finitely additive $G$-invariant measure. For example, $X$ could be a nonamenable group and $G=X$. Then by 
Tarski's Theorem [Pat, 3.15], $X$ is $G$-paradoxical. That is, there exists a partition $A_{1}, \ldots, A_{m}, B_{1}, \ldots, B_{n}$ of $X$ and elements $x_{1}, \ldots, x_{m}, y_{1}, \ldots, y_{n}$ of $\Gamma$ such that $\left\{x_{1} A_{1}, \ldots, x_{m} A_{m}\right\}$ is a partition of $X$ and $\left\{y_{1} B_{1}, \ldots, y_{n} B_{n}\right\}$ is a partition of $X$. Let $K=\left\{x_{1}, \ldots, x_{m}, y_{1}, \ldots, y_{n}\right\}$, and consider the group $\langle K\rangle$ generated by $K$. Let $\pi$ denote the quasi-regular representation of $\langle K\rangle$ on $l^{2}(X)$, that is,

$$
\pi(g) f(x)=f\left(g^{-1} x\right), \quad g \in\langle K\rangle, f \in l^{2}(X), x \in X .
$$

Proposition 9. $\kappa(\pi, K) \geq \frac{1}{2(m+n)}$.

Proof. For a subset $S$ of $X$ let $E_{S}$ denote the orthogonal projection of $l^{2}(X)$ onto $l^{2}(S)$, that is,

$$
E_{S} \xi(g)= \begin{cases}\xi(g) & \text { if } g \in S \\ 0 & \text { otherwise }\end{cases}
$$

Direct calculation shows that $\pi(g) E_{S} \pi\left(g^{-1}\right)=E_{g S}$. $n)$.

Let $v_{i}=\pi\left(x_{i}\right), e_{i}=E_{A_{i}}(1 \leq i \leq m)$ and $w_{j}=\pi\left(y_{j}\right), f_{j}=E_{B_{j}} \quad(1 \leq j \leq$

Then we can write the paradoxical decomposition in terms of bounded operators on $l^{2}(X)$ as follows:

$$
1=\sum e_{i}+\sum f_{j}=\sum v_{i} e_{i} v_{i}^{*}=\sum w_{j} f_{j} w_{j}^{*} .
$$

Let $\varepsilon=\kappa(\pi, K)$, and let $\xi \in l^{2}(X)$ with $\|\xi\|=1$. Then

$$
\left\|v_{i} \xi-\xi\right\| \leq \varepsilon, \quad\left\|w_{j} \xi-\xi\right\| \leq \varepsilon .
$$

Hence, also

$$
\left\|v_{i}^{*} \xi-\xi\right\| \leq \varepsilon, \quad\left\|w_{j}^{*} \xi-\xi\right\| \leq \varepsilon
$$

for $1 \leq i \leq m, 1 \leq j \leq n$.

Now $\varepsilon \geq\left\|e_{i}\left(v_{i}^{*} \xi-\xi\right)\right\| \geq\left|\left\|e_{i} v_{i}^{*} \xi\right\|-\left\|e_{i} \xi\right\|\right|$. Therefore,

$$
\left|\left\|e_{i} v_{i}^{*} \xi\right\|^{2}-\left\|e_{i} \xi\right\|^{2}\right|=\left|\left\|e_{i} v_{i}^{*} \xi\right\|+\left\|e_{i} \xi\right\|\right|\left|\left\|e_{i} v_{i}^{*} \xi\right\|-\left\|e_{i} \xi\right\|\right| \leq 2\|\xi\| \varepsilon \leq 2 \varepsilon
$$

and so $\left\|e_{i} v_{i}^{*} \xi\right\|^{2} \leq\left\|e_{i} \xi\right\|^{2}+2 \varepsilon$. Similarly $\left\|f_{j} w_{j}^{*} \xi\right\|^{2} \leq\left\|f_{j} \xi\right\|^{2}+2 \varepsilon$. It follows that

$$
1=\|\xi\|^{2}=\sum_{i}\left\langle v_{i} e_{i} v_{i}^{*} \xi \mid \xi\right\rangle=\sum_{i}\left\|e_{i} v_{i}^{*} \xi\right\|^{2} \leq \sum_{i}\left\|e_{i} \xi\right\|^{2}+2 m \varepsilon
$$

and similarly

$$
1 \leq \sum_{j}\left\|f_{j} \xi\right\|^{2}+2 n \varepsilon
$$

Now $1=\sum e_{i}+\sum f_{j}$. Therefore,

$$
1=\sum_{i}\left\|e_{i} \xi\right\|^{2}+\sum_{j}\left\|f_{j} \xi\right\|^{2} \geq 1-2 m \varepsilon+1-2 n \varepsilon,
$$

that is, $2(m+n) \varepsilon \geq 1$, as required. 


\section{REFERENCES}

[AkW] C. A. Akemann and M. E. Walter, Unbounded negative definite functions, Canad. J. Math. 33 (1981), 862-871.

[Del] P. Delorme, 1-cohomologie des représentations unitaires des groupes de Lie semisimples et résolubles. Produits tensoriels et représentations, Bull. Soc. Math. France 105 (1977), 281-336.

[Gui] A. Guichardet, Étude de la 1-cohomologie et la topologie du dual pour les groupes de Lie à radical abélien, Math. Ann. 228 (1977), 215-232.

[HaV] P. de la Harpe and A. Valette, La propriété (T) de Kazhdan pour les groupes localement compacts, Astérisque 175 (1989).

[HRV] P. de la Harpe, A. G. Robertson, and A. Valette, On the spectrum of the sum of generators for a finitely generated group, Israel J. Math. 81 (1993), 65-96.

[KaT] E. Kaniuth and K. F. Taylor, Kazhdan constants and the dual space topology, preprint, Paderborn, 1992.

[Pat] A. L. T. Paterson, Amenability, Math. Surveys Monographs, vol. 29, Amer. Math. Soc., Providence, RI, 1988.

[Sak] S. Sakai, $C^{*}$-algebras and $W^{*}$-algebras, Springer-Verlag, New York, 1971.

Institut de Mathématiques, Chantemerle 20, 2007 Neuchâtel, SWitzerland

Current address: c/o 12 Langton Avenue, London N20 9DB, England

Department of Mathematics, University of Newcastle, New South Wales 2308, AusTRALIA

E-mail address: guyanefrey.newcastle.edu. au 